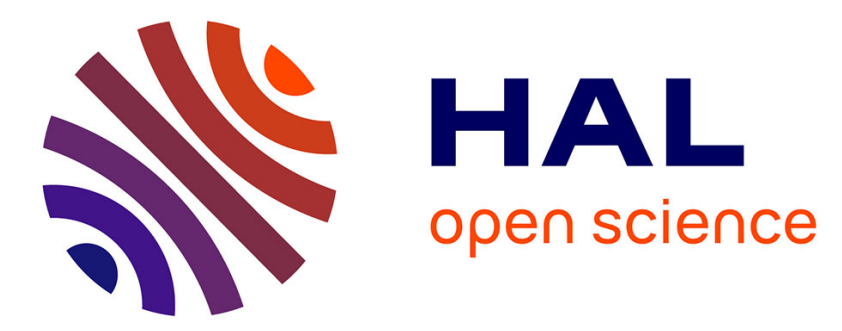

\title{
Ce que le dialogue social fait au sport
}

\author{
Sébastien Fleuriel
}

\section{To cite this version:}

Sébastien Fleuriel. Ce que le dialogue social fait au sport. Sciences sociales et sport, 2017, 10, https://www.cairn.info/revue-sciences-sociales-et-sport-2017-1-p-141.htm. $\quad$ 10.3917/rsss.010.0141 . halshs-01536366

\section{HAL Id: halshs-01536366 \\ https://shs.hal.science/halshs-01536366}

Submitted on 13 Jun 2017

HAL is a multi-disciplinary open access archive for the deposit and dissemination of scientific research documents, whether they are published or not. The documents may come from teaching and research institutions in France or abroad, or from public or private research centers.
L'archive ouverte pluridisciplinaire $\mathbf{H A L}$, est destinée au dépôt et à la diffusion de documents scientifiques de niveau recherche, publiés ou non, émanant des établissements d'enseignement et de recherche français ou étrangers, des laboratoires publics ou privés. 


\section{Ce que le dialogue social fait au sport}

\section{Sébastien Fleuriel, professeur de sociologie, CLERSE Université Lille 1}

«Et la convention collective, c'était un registre neuf par rapport à celui qu'il fallait quitter, et c'était un registre sur lequel on pouvait peut-être espérer faire quelque chose. » (Négociateur de la branche professionnelle du sport, entretien du 21 mars 2013)

L'année 2005 formalise à travers la convention collective nationale du sport (CCNS) un processus de régulation du travail dans la branche professionnelle dite du sport, entamé au tournant des années 1990. Participant du système des relations professionnelles entendues comme « relations qui s'établissent entre trois catégories d'acteurs, les pouvoirs publics, les salariés et leurs représentants, les employeurs et leurs organisations $»^{1}$, ce processus s'inscrit plus généralement dans l'histoire des modes de négociation et de régulations des conflits au travail normalisée en France dès l'orée du $20^{\mathrm{e}}$ siècle par la production juridique de la convention collective dans la loi du 4 mars $1919^{2}$. Il résulte, plus récemment encore, de la volonté de la classe politique d'élever significativement, à la suite des lois Auroux de 1982, le taux de couverture conventionnelle de tous les secteurs du travail, pour le porter de $75 \%$ en 1982 à 97 $\%$ aujourd'hui ${ }^{3}$. Le sport, considéré ici comme secteur d'emplois, longtemps resté en marge de ce mouvement, s'est ainsi vu très fortement incité en 1990 à rejoindre cette dynamique en se plaçant sous le sceau d'une convention collective ad hoc ou, à défaut, intégrer une convention déjà existante ${ }^{4}$.

\footnotetext{
${ }^{1}$ BeVORT, Antoine ; JOBERT, Annette. 2008, Sociologie du travail: les relations professionnelles, Paris, France, A. Colin, p. 3.

${ }^{2}$ Voir DIDRY, Claude. 2002. Naissance de la convention collective: débats juridiques et luttes sociales en France au début du 20e siècle, Paris, École des hautes études en sciences sociales, 267 p.

${ }^{3}$ Ces données sont fournies par BECHAUX Stéphane. 2013. «Dans la jungle des conventions collectives », Liaisons Sociales, 138, p. 26-29. Une histoire plus générale des conventions collectives en France est astucieusement synthétisée par TALLARD Michèle. 2004. «Conventions collectives et hiérarchies des qualifications », in MEDA Dominique; VenNAT Francis, (dirs.). 2004. Le travail non qualifié: permanences et paradoxes, Paris, La Découverte, p. 41-54.

${ }^{4}$ Jean-Pierre Soisson, alors Ministre du travail, s'adresse notamment dans un courrier au président du CNOSF dans ces termes: "Or, le sport est l'un des derniers grands secteurs à ne pas être couvert pour une ou des conventions de branche, et ce, malgré la politique menée sans discontinuité depuis 1982 en plein accord avec les partenaires sociaux réunis au sein de la Commission Nationale de la Négociation collective pour généraliser la couverture conventionnelle à toutes les branches [...] Le sport doit participer à cette évolution fondamentale... », Lettre du Ministre du Travail, Jean-Pierre Soisson au président du CNOSF, Nelson Paillou, du 6 février 1990 (archive privée de l'UNSA).
} 
Fortement suggérée par le haut depuis la Direction générale du travail du Ministère éponyme, la CCNS sollicite, dès les premières négociations, un ensemble conséquent de nouveaux acteurs qui doivent assurer une représentativité aussi fidèle que possible des employeurs et des employés concernés par les pratiques sportives.

En plus des traditionnelles centrales syndicales présumées irréfragables de représentativité ${ }^{5}$, la CCNS contraint les dirigeants sportifs à se constituer de toute pièce en organisation représentative des employeurs. Le Conseil social du mouvement sportif (CoSMoS), distinct des organes traditionnels de représentation des intérêts sportifs tels que le CNOSF ou les fédérations $^{6}$, est appelé à partager son mandat avec les employeurs de l'animation socioculturelle, eux aussi massivement concernés par l'emploi sportif et regroupés autour de l'économie sociale et solidaire ${ }^{7}$. Au total, ce sont initialement 8 organisations de salariés et 4 organisations d'employeurs qui participent ainsi à l'écriture d'un droit conventionnel propre au sport $^{8}$.

Les délégués du CoSMoS, dirigeants traditionnels du mouvement sportif convertis en représentants d'une partie des employeurs du sport, sont ainsi amenés à côtoyer dans une nouvelle arène, désignée en tant que branche professionnelle du sport, de nouveaux acteurs syndicaux et patronaux. Chargés tous ensemble de procéder à une forme de normalisation du travail sportif, entendue ici comme processus tardif de mise en conformité des activités sportives avec le droit du travail ou le droit commun ${ }^{9}$, ces acteurs de statuts et de trajectoires diversifiés, doivent interagir dans un cadre nouveau, en y important ou prolongeant les

\footnotetext{
${ }^{5}$ En France jusqu'en 2008, seuls certains syndicats (CGT, CGT-FO, CFDT, CFTC, CFE-CGC) étaient considérés comme représentatifs et bénéficiaient d'une présomption irréfragable de représentativité. Ils n'avaient donc pas à prouver leur représentativité, qui était présumée dans l'arrêté du 31 mars 1966, complétant une décision du 8 avril 1948. La loi du 20 juin 2008 supprime la représentativité irréfragable et la redéfinit en 7 critères: le respect des valeurs républicaines, l'indépendance, la transparence financière, une ancienneté minimale de deux ans dans le champ professionnel et géographique couvrant le niveau de négociation, l'influence (caractérisée par l'activité et l'expérience), les effectifs d'adhérents et les cotisations, l'audience établie selon les niveaux de négociation.

${ }^{6}$ Nous revenons plus bas sur cette nécessité de distinguer le CNOSF du CoSMoS.

${ }^{7}$ FLEURIEL, Sébastien. 2013. «Ce que dialoguer veut dire. L'émergence du «dialogue social» dans le sport français (1990-2006) », Genèses, 92, p. 127-146.

${ }^{8}$ A l'origine, six organisations salariées, FO, la CFDT, la CFTC, la CGC, la Confédération nationale des éducateurs sportifs (CNES), la Fédération nationale des associations et syndicats de sportifs (FNASS) sont signataires de l'accord le 7 juillet 2005, l'UNSA et la CGT ont refusé la signature mais participent aux négociations de branche. Pour les organisations patronales, le CoSMoS, et le Conseil national des employeurs associatifs (CNEA) sont signataires. Le CNEA résulte pour sa part du regroupement réalisé à la veille de la signature, des trois syndicats qui ont participé à toutes les négociations en tant qu'entité propre : le Syndicat national des organisations gestionnaires d'activités éducatives et culturelles (SNOGAEC), le Syndicat des associations de développement culturel et social (SADCS) et l'Union nationale des organismes de développement social, sportif et culturel (UNODESC).

${ }^{9}$ Il faudrait en toute rigueur préciser que le travail sportif n'est pas limité aux seuls sportifs dits ou reconnus professionnels mais qu'il englobe également l'ensemble des travailleurs qui exercent une activité professionnelle en lien avec le sport, notamment l'ensemble des moniteurs et éducateurs sportifs.
} 
préoccupations et catégories d'analyse déjà en vigueur dans le champ sportif ${ }^{10}$. Agissant comme une chambre d'écho des divisions et des luttes qui traversent usuellement l'espace des sports, la récente branche professionnelle contribue alors à déplacer ces questions dans un univers nouveau référé au droit du travail autant qu'à l'ordre sportif constitué de ses règles propres (réglementations et juridictions sportives spécifiques, calendriers compétitifs particuliers, organisations sportives autonomisées, etc.) En retour, la production d'un ordre normatif conventionnel a par définition vocation à agir sur les comportements des intéressés, employeurs et employés, et ne reste pas sans effet sur les conduites observées dans le champ en imposant une réglementation du travail spécifique. Il s'agit dès lors de comprendre dans quelles mesures cette activité, imparfaitement résumée sous le vocable de «dialogue social ${ }^{11}$, participe des rapports de force et d'opposition constatés dans le champ sportif. Cette scène particulière, peu visible dans les médias, reste également soustraite aux analyses sociologiques sur le champ sportif alors qu'elle concourt à sa structuration en tant qu'elle se trouve dotée d'un pouvoir normatif effectif ${ }^{12}$.

Appréhender de quelle manière les luttes internes pour la définition légitime du sport dans leur dynamique générale, se sont répercutées dans les débats soulevés par l'émergence du dialogue social dans le sport implique de revenir sur la manière dont « les acteurs impliqués [...] ont débattu de l'architecture conventionnelle du sport face aux règlements fédéraux d'une part, et aux principales évolutions législatives et jurisprudentielles du droit du travail de l'autre $»^{13}$. Il s'agit alors de faire porter les efforts d'analyse sur la façon dont les catégories en usage dans le champ ont été reprises et retraduites dans la CCNS pour s'imposer finalement comme des normes de droit applicables à l'ensemble. On ne cherchera donc pas ici à vérifier jusqu'à quel point ces normes sont effectivement opératoires et agissantes auprès des acteurs jusque dans leurs pratiques effectives, sachant que des travaux en cours font le constat d'une lente

\footnotetext{
${ }^{10}$ Bien qu'on ait pleinement conscience du caractère réducteur de l'expression, on parlera par commodité de champ sportif tout au long du texte. Sur ces deux formes possibles de désignation, voir DeFRANCE, Jacques. 1995. «L'autonomisation du champ sportif. 1890-1970 », Sociologie et sociétés, vol. 27, n¹, p. 15-31 et DeFranCE, Jacques. 2012. «L'autonomie d'un champ ou le sens des limites », in LEBARON, Frédéric ; MAUGER, Gérard (dirs.), Bourdieu, Paris, Ellipses, p. 319-349.

${ }^{11}$ Sur le sens à donner à cette expression, lire Higele, Jean-Pascal. 2012. "Les formes de la délibération interprofessionnelle. Le sens du dialogue », Sociétés contemporaines, 86, p. 85-111.

${ }^{12}$ C'est plus généralement la question du travail et de l'emploi dans le sport qui s'avère encore peu explorée. Quand elle l'est, c'est le plus souvent quand les sociologues cherchent à faire œuvre de déconstruction du couple d'oppositions amateur/professionnel ou pour penser la « professionnalisation » du sport en général.

${ }^{13}$ BuIsINE, Sébastien. 2009. «Le processus d'harmonisation du dialogue social dans le sport professionnel », Terrains \& travaux, 14, p. 109-126.
} 
intégration sur le terrain ${ }^{14}$, et que de attentes fortes s'expriment plus explicitement aujourd'hui qu'hier $^{15}$. Le raisonnement consiste ici plutôt à focaliser sur la manière dont un ensemble de catégories pratiques en vigueur dans le champ sportif, et qui contribue à définir le sport, telles que amateur/professionnel, loisir/compétition, marchand/non marchand, etc., a pu se trouver questionné, voire déstabilisé, par la prise en charge du travail dans le sport par le droit conventionnel.

L'analyse s'inspire d'un premier travail d'observation, commencé en 2012, des négociations de la branche sport en séances plénières dites commissions mixtes paritaires (soit 26 demijournées), dont la vocation consiste à réunir «représentants patronaux et syndicaux d'une profession donnée sous l'égide d'un agent de l'Etat, le plus souvent un directeur ou inspecteur du travail, dans le but de créer ou de réformer une convention collective. ${ }^{16}$ Constituées en autant de scènes où les acteurs tiennent le jeu de rôles en conformité avec les organisations qu'ils représentent, ces séances forment une excellente chambre d'échos des débats en vigueur et surtout de la manière dont les acteurs appréhendent et pensent les problèmes qu'ils se posent. En reprenant la formule de Jean Saglio qui constatait qu'au-delà des oppositions de principe, «les protagonistes sont d'accord sur un point, à savoir que c'est entre eux que cela va se jouer $»^{17}$, on peut ajouter que ceux-ci partagent les mêmes catégories d'analyse de la réalité qu'ils cherchent à travailler. Selon une procédure relativement ritualisée, les négociateurs indiquent sur les sujets portés à l'ordre du jour (revalorisation des salaires, prévoyance, égalité femme/homme, formation professionnelle,...) les positions que leur organisation défend, et apprécient jusqu'à quel point des marges de manœuvre sont susceptibles de déboucher à une position majoritaire. A ces positions officielles et rendues publiques en réunion selon un jeu quasi théâtral qui confine parfois à la caricature, répondent des interruptions de séance lorsque le consensus semble impossible, ouvrant alors la voie à des négociations off, des réajustements,

\footnotetext{
${ }^{14}$ DeVETTER, François-Xavier ; FLEURIEL, Sebastien. 2016. « Les relations sociales dans le sport : un processus inachevé », Marché et organisations, n²7, p. 45-57.

${ }^{15}$ Lire par exemple BARBUSSE, Béatrice. 2012. « Les conditions sociales d'émergence d'une nouvelle gouvernance des sports collectifs professionnels en France : le cas du handball masculin », in ATTALI, Michaël ; BAZOGE, Natalia (dirs.), Diriger le sport: perspectives sur la gouvernance du sport du XXe siècle à nos jours, Paris, CNRS éd., p. 261-276.

${ }^{16}$ BEVORT, Antoine ; JOBERT, Annette. 2008, op. cit., p. 83. Les auteurs ajoutent en outre que « Le représentant de l'administration préside les séances, assure le respect des procédures, donne des informations sur le contenu de la législation, sans pourtant «dire le droit » au sens où le juge tranche un litige et donne raison à l'une des parties contre l'autre. Ni juge, ni arbitre, son rôle est plutôt celui d'un animateur, un « facilitateur » de la négociation.

${ }^{17}$ SAGLIO, Jean. 2006. « Les relations professionnelles face aux nouveaux rapports de subordination », dans PETIT, Héloïse ; THEVENOT, Nadine (dirs.), Les nouvelles frontières du travail subordonné: approche pluridisciplinaire, Paris, France, la Découverte, p. 242.
} 
des explicitations des enjeux ou intérêts cachés. Témoin de la quasi intégralité de ces moments depuis plusieurs années, j'ai ainsi pu collecter un matériau de première main qui rassemble aussi bien le contenu des débats publics et leurs actes officiels (ordres du jour, compte-rendu de séances et documents préparatoires) que le contenu off des discussions informelles faisant état des rapports de force, des moyens de pression, et des lectures indigènes relatives aux divers blocages constatés. L'accès à ces sources est par ailleurs étroitement conditionné à la connaissance d'un informateur privilégié représentant d'une organisation salariée et régulièrement disposé à m'initier à l'actualité conventionnelle. Outre ce matériau, la collecte s'enrichit d'une campagne d'entretiens semi-directifs $(n=25)$ menés auprès des négociateurs passés ou actuels de toutes les organisations, employeurs comme employés, impliquées dans la branche $^{18}$, afin d'éclaircir outre des éléments de trajectoire sociale, les conditions d'exercice de leur mandat vis-à-vis de leur organisation et la légitimité qu'ils prétendent tirer de leurs adhérents sur les questions proprement sportives. La réalisation progressive de ces entretiens menés en marge des négociations a ainsi pu contribuer à asseoir graduellement ma présence d'une commission à l'autre et à m'installer dans le paysage comme un observateur en position d'écoute attentive. Enfin, un travail sur archives mené auprès de deux sources distinctes, les archives nationales d'une part et celles de la CCNS d'autre part, a cherché à contrôler l'histoire et l'évolution de cette convention collective à travers les échanges entretenus entre les services respectifs des ministères du travail, du sport, les fédérations et le CNOSF sur la question des relations professionnelles, révélant là encore, les catégories par lesquelles le sport est appréhendé dans sa relation au travail.

Partant d'une exploitation ciblée de ces matériaux, cet article s'attache dans un premier temps à montrer comment la problématique du travail dans le sport et de son absence d'encadrement juridique a pu apparaitre révélatrice des profonds clivages qui traversent l'ordre sportif. Il s'agira de montrer dans un second temps pourquoi la CCNS, apparue dans ces conditions comme une réponse crédible et opportune pour résoudre ces tensions, s'est alors faite caisse de résonnance de celles-ci en réveillant de coûteux débats sur les catégories pratiques mobilisées usuellement dans le sport. Enfin, l'analyse portera dans un troisième temps sur les effets qu'exerce en retour le traitement de ces débats sur l'ordre sportif.

\footnotetext{
${ }^{18}$ Notamment avec les représentants de FO ( $\left.n=2\right)$, CFDT ( $\left.n=3\right)$, CGT ( $\left.n=1\right)$, CFE-CGC ( $\left.n=2\right)$, CFTC ( $\left.n=1\right)$, FNASS $(n=2)$, UNSA $(n=2)$, UNODESC $(n=1), \operatorname{SNOGAEC}(n=1), \operatorname{CNEA}(n=4), \operatorname{CoSMOS}(n=3)$, DGT du Ministère du travail $(n=3)$.
} 


\section{I - La normalisation du travail sportif révélatrice de profonds clivages dans le sport}

C'est au milieu des années 1990 que l'injonction du Ministère du travail pour assurer la couverture conventionnelle du secteur sportif se fait la plus pressante en proposant fin 1996 et faute d'accord explicite, de l'intégrer à la convention collective de l'animation socioculturelle. Cette menace, dont certains observateurs se demanderont si elle n'a pas été expressément souhaitée par les dirigeants du CNOSF pour créer l'électrochoc propice à une mobilisation fédérale qui tarde à venir ${ }^{19}$, a pour effet quasi immédiat de déboucher sur la création du CoSMoS en janvier 1997 puis, dans la foulée, sur l'ouverture des premières négociations entre organisations patronales pour définir le champ d'application de la convention collective à $v^{v e n i r}{ }^{20}$. Cette période coïncide avec l'installation au Ministère de la jeunesse et des sports de Marie-George Buffet $^{21}$ dont la préoccupation principale consiste à réaffirmer l'unité du mouvement sportif en proie notamment à un écartèlement rédhibitoire entre sport amateur et sport professionnel. Maniant à l'envi les catégories d'analyse en usage dans le champ sportif, le discours que la Ministre des sports prononce devant ses homologues de l'Union Européenne est caractéristique des préoccupations françaises et en traduit significativement les enjeux internes : le public y est opposé au privé, le désintéressement à l'argent, le bénévolat à la professionnalisation, l'associatif aux forces du marché,... (Voir encadré 1 : les enjeux internes au champ sportif).

\footnotetext{
${ }^{19}$ Dans un premier temps, les dirigeants des fédérations ne voient guère l'intérêt d'adopter une convention collective. On peut en effet lire dans une note de la sous-direction de la négociation collective du Ministère du travail et affaires sociales, adressée à son directeur, Jean Marimbert, datée du 18 octobre 1995 le constat suivant : «Le CNOSF se demande si la «menace» de l'animation socio-culturelle ne peut pas être le «coup de pouce » qui l'aiderait à convaincre ses fédérations sportives à s'organiser en organisations d'employeurs ». Archives Nationales, cote 20111081/68.

${ }^{20}$ La définition du champ d'application d'une convention collective est en effet une prérogative exclusive des organisations patronales. Dans la même note de synthèse de la sous-direction de la négociation collective, il est souligné que "la reconnaissance du CNOSF comme organisation d"employeurs se heurtait à de sérieuses difficultés juridiques : [dans la mesure où] - ses statuts ne comportent pas d'objet social spécifique ; - le CNOSF représente l'ensemble des sportifs et du mouvement sportif, et pas uniquement les employeurs. Par ailleurs il bénéficie d'un monopole de représentation, assorti d'un système d'adhésion obligatoire ; - il est attributaire de prérogatives de puissance publique comportant notamment un pouvoir disciplinaire. La seule solution juridiquement solide serait pour le CNOSF de susciter la création d'une association d'employeurs dotée d'un mandat social explicite. » AN cote 20111081/68. Le CNOSF n'ayant donc pas eu l'autorisation de se constituer en organisation représentative des employeurs, et par ailleurs l'animation socio-culturelle ne rassemblant quasi aucun dirigeant fédéral ; le CoSMoS est ainsi créé le 23 janvier 1997 pour répondre à cette impasse juridique. Ces conditions contribuent à rendre cette organisation centrale dans le processus de négociations en tant représentative des employeurs situés au cœur du champ sportif.

${ }^{21}$ Sur les caractéristiques de ce Ministère, lire MARTINACHE, Igor. 2013. «Le Ministère Buffet (1997-2002) : contraintes et contradictions dans la mise en œuvre d'une politique sportive de gauche », Cahiers d'histoire. Revue d'histoire critique, 120, p. 103-115.
} 


\section{Encadré 1 : les enjeux internes au champ sportif}

Le contexte des années 2000 fait coïncider sur l'agenda de la Ministre des sports en exercice, Marie-George Buffet, les discussions sur la constitution de la convention collective nationale du sport et la sauvegarde d'une conception du sport « à la française » dans l'Union Européenne. S'adressant aux ministres des sports de l'UE, le discours de Marie-George Buffet donne à percevoir un florilège paradigmatique des préoccupations propres au champ sportif ainsi qu'à leur traduction indigène où les valeurs s'opposent au marché, le public au privé, la professionnalisation au bénévolat, la masse à l'élite, l'associatif aux autres formes de groupement, etc. L'exercice auquel se livre la Ministre est aussi celui du maintien de l'unité et de la cohérence du «mouvement sportif » par-delà ces mêmes divisions.

«Le sport, en effet, mobilise chaque semaine des dizaines de milliers de bénévoles, rassemble des millions de pratiquants, et fait rêver les hommes et les femmes de nos pays. [...] Le sport a acquis une dimension sociétale, à la fois de proximité, mais également planétaire. [...] Le sport est en effet confronté à des enjeux considérables liés à son développement, à la diversification des pratiques, mais aussi à sa médiatisation, à sa professionnalisation, à l'arrivée massive d'argent dans certaines disciplines. [...]Vous savez que je n'ai pas la volonté de diaboliser l'argent dans le sport. Le sport a besoin de moyens et ces moyens ne peuvent pas être uniquement publics. Je me refuse ainsi à délimiter un sport sans argent qui serait " pur " et un sport bénéficiant d'argent qui serait " perdu ".Je préfère poser la question autrement : le mouvement sportif dispose-t-il des moyens, des espaces, des bases légales pour préserver le sens, les valeurs du sport en maîtrisant l'argent qui lui arrive et ainsi éviter que cet argent dicte sa loi au sport?

Deux choix sont possibles. Soit à partir du constat de ces dérives, nous considérons que le sport ne dépend plus que du marché, les sportifs de leur valeur ajoutée ; alors, il faut leur appliquer les règles de la concurrence, et ajouter de la déréglementation aux folies actuelles.

Soit, à partir de ce que fut la construction du sport moderne en Europe, avec ses valeurs humanistes, ses structures associatives, nous décidons, dans le contexte actuel, de redonner en Europe aux mouvements sportifs les moyens juridiques et institutionnels de protéger les pratiques sportives dans leur diversité au sein des structures fédératives.

Cette dernière option, qui est celle de la présidence française, [...] En ce qui concerne les pouvoirs publics je crois qu'il est de notre responsabilité de créer les conditions pour que ces associations sportives, autonomes par essence, puissent mener à bien leurs missions sans voir leur rôle remis en cause par des groupes extérieurs au monde associatif, et dont les objectifs sont d'utiliser le sport à des fins d'intérêts privés. »

Source : Extraits de la déclaration de Mme Marie-George Buffet, ministre de la jeunesse et des sports, sur la politique européenne du sport notamment, la nécessité de préserver les valeurs du sport face aux dérives mercantiles et aux règles de la concurrence, Paris le 6 novembre 2000.

Au tournant des années 2000, ces luttes internes cristallisent donc de fortes oppositions entre les dirigeants des fédérations sportives qu'aucun discours de surplomb, fût-il rassembleur, ne parvient à faire taire. Ce n'est pas un hasard si elles rencontrent une forte résonnance dans le contexte d'un dialogue social en émergence et sommé de prendre en charge la question du travail dans le sport. A côté des débats officiels sur le sport professionnel, se jouent dans 
l'antichambre du CNOSF et du CoSMoS dont les intérêts sont au départ indissociables ${ }^{22}$, des rapports de force visant à freiner autant que faire se peut les aspirations des fédérations sportives, notamment celles qui disposent d'un secteur professionnel, à fixer leurs propres cadres référentiels de travail, et ce en relative indépendance. La charte du football professionnel adoptée en 1973 ouvre dans cette perspective la voie d'une régulation du travail conçue sport par sport de façon relativement étanche, régulation qui conduit à l'adoption d'une convention collective dans l'équitation en 1975, une convention pour les personnels administratifs du football en 1983, une autre pour le golf en 1995. Conventions auxquelles viennent s'ajouter progressivement des accords dits sectoriels dans le rugby, le basketball, le handball, le cyclisme, sans compter les velléités des maitres de nageurs sauveteurs en $1996^{23}$ puis celles des haltérophiles en $1997^{24}$ à constituer leur propre convention.

D'abord enjoint à se positionner contre l'animation socioculturelle, une large fraction des dirigeants sportifs doit faire face à des forces puissamment centrifuges qui poussent les disciplines sportives et leurs organisations fédérales à élaborer en relative indépendance leurs propres règles du jeu en matière de travail. Alignés sur les aspirations unificatrices de la Ministre des sports, les dirigeants du CNOSF et de son émanation patronale le CoSMoS, s'attèlent alors à jouer les rassembleurs universels contre les intérêts particuliers, ou encore selon leurs propres mots contre «les petites chefferies» ou «réflexes individualistes des fédérations » peu disposées à jouer collectif, à l'image du football professionnel, dont les dirigeants de l'union des clubs professionnels de football (UCPF) œuvrent en arrière-plan pour échapper à la convention collective du sport en cours de négociation, comme le rappelait sans détour en entretien son directeur général du moment (et toujours en exercice) :

J'ai dit « voilà, encore une fois, on trouve que c'est très bien que vous ayez une structuration du dialogue social et une convention collective du sport, mais nous, on n'a pas besoin d'un texte supplémentaire, puisqu'on a déjà une convention du football qui date de 1973, on a été très (insiste sur le terme) dans l'anticipation par rapport à tous ces phénomènes, qui fonctionne très bien, avec plein de partenaires identifiés, donc

\footnotetext{
${ }^{22}$ Son premier président, François Alaphilippe, fut aussi et d'abord le plus jeune secrétaire général du CNOSF nommé à cette fonction (mai 1993). On peut supposer qu'il a à cœur, en tant que nouvel entrant, de conduire une réforme efficace de son organisation vis-à-vis du travail. Il est contraint de démissionner afin de pouvoir se consacrer exclusivement à la présidence du CoSMoS.

${ }^{23}$ Lettre de J.-M. Lapoux, secrétaire général de la Fédération des maîtres-nageurs sauveteurs, 8 octobre 1996, archives de la CCNS déposées au CoSMoS.

${ }^{24}$ Note de Michel Popov, «Élaboration d'une convention collective pour les métiers du sport dans les Hauts-deSeine à partir des pratiques de l'haltérophilie-musculation », 11 décembre 1996, archives de la CCNS déposées au CoSMoS.
} 
sortez nous du champ ! ». Et je dois avouer, encore une fois que le Ministère du travail était convaincu de ça. Qu'il souhaitait et qu'il était prêt à nous sortir du champ. (Entretien du 9 décembre 2014.)

En dépit de l'unité du mouvement sportif proclamée par certains acteurs, dont les dirigeants du CNOSF, qui ont un intérêt stratégique à se poser en rassembleurs, le champ sportif est en réalité traversé par de fortes dissensions qui opposent durablement les représentants des disciplines sportives entre eux en matière de régulation du travail, les faisant agir en ordre dispersé selon des intérêts propres. C'est dans ce contexte que la CCNS, comme instance normalisatrice supposée homogénéiser l'ensemble de ces régulations, se fait alors caisse de résonnance de toutes les dissensions sportives.

\section{II - La CCNS comme caisse de résonnance des dissensions sportives}

Bien qu'initialement assez peu enthousiastes pour se doter d'une couverture conventionnelle, les dirigeants du CNOSF perçoivent finalement en celle-ci, sous les fortes pressions du Ministère du travail, une opportunité de faire tenir ensemble toutes les forces divergentes qui tirent dans des directions opposées. Perçue comme le ciment qui manquait jusqu'ici pour amalgamer le tout, la CCNS prend un intérêt nouveau au regard des risques latents de dispersion. Le secrétaire général du CNOSF revient ainsi sur les intentions qui ont amené son organisation à s'engager dans cette voie :

L'intérêt [d'une convention] pour le mouvement sportif, c'était un élément d'unité. Une unification. Une manière de lier les énergies. Je vous disais toute à l'heure que chacun restait regroupé sous sa guitoune, comment voulez-vous, à partir de là, mûrir ? Il devait y avoir une situation, je dirais, politiquement ou socialement plus affirmée, quand chacun reste dans son coin. Le danger, c'est que cette division se démultiplie encore avec la montée du sport professionnel, avec que sais-je encore ? Des tas de phénomènes qui font que chacun reste dans sa boutique. Et alors là, c'est pas la peine d'espérer peser sur quoi que ce soit, dans le système de l'Etat, et même dans le système sportif mondial. Il faut essayer de s'affirmer, d'affirmer une unité, on a tous à y gagner. Et la convention collective, c'était un registre neuf par rapport à celui qu'il fallait quitter, et c'était un registre sur lequel on pouvait peut-être espérer faire quelque chose. (Entretien du 21 mars 2013.) 
Envisagées dans une stratégie de resserrement à un moment perçu comme critique, les premières négociations soulèvent cependant des questions complexes à l'ordre sportif, à commencer d'abord par la définition-même du sport. La formation d'une branche professionnelle et de la convention collective qui lui est rattachée, implique en effet de fixer irrémédiablement les délimitations de son champ d'application, selon des prérogatives exclusivement exercées par les organisations patronales. La coïncidence de vocabulaire entre champ sportif et champ d'application n'est ici pas qu'une affaire de sémantique, elle suppose de déterminer clairement qui prétend encadrer le sport et qui ne peut y prétendre, c'est-à-dire quelles sont les organisations pouvant s'affirmer représentatives des pratiques sportives (voir encadré 2 : le champ d'application de la CCNS, quelle définition du sport ?) Les lignes de clivage s'organisent d'une part entre ce qui relève de l'animation socioculturelle et de l'animation sportive; et d'autre part, entre ce qui dépend des pratiques commerciales et marchandes et les activités non marchandes. Mais à la manière d'un jeu de poupées russes, ces clivages renvoient secondairement à d'autres couples d'opposition qui ne se recoupent jamais très exactement entre structures associatives et entreprises, pratiques de loisir et compétitions, amateurisme et professionnalisme ${ }^{25}, \ldots$, les critères discriminants relevant tantôt de formes organisationnelles ou juridiques, tantôt des formes d'activités, voire combinant les deux. Un négociateur patronal ayant participé aux premières discussions sur les contours du champ d'application explique par exemple :

Alors après, l'autre élément, et là le clivage était en effet... il n'était pas entre guillemets entre acteurs du sport et acteurs de l'animation, il était beaucoup beaucoup plus vaste, puisque c'est soit avoir tout le sport, soit uniquement le sport associatif. Moi, à titre personnel, j'étais favorable, même s'il y avait deux conventions collectives, avec une convention collective associative qu'on ne mélange pas au sport professionnel et commercial. [...]Et ça, le comité olympique n'en voulait pas et Marie-George Buffet a pris position dans ce sens, bon elle a eu un certain nombre de conseillers. Mais ça, ça a été tranché politiquement : une seule convention collective même en sachant que ça serait ingérable ! (insiste sur le terme) Et ça, on le voit aujourd'hui. Donc elle est unique, pour tout le sport, qu'il soit en effet associatif, à but non lucratif, commercial.

\footnotetext{
${ }^{25}$ Un contenu plus détaillé de la définition conventionnelle du sport professionnel par apposition au sport amateur est donné dans : FLEURIEL S., 2016, «Le sport professionnel saisi par sa convention collective : genèse d'une définition singulière », Le Mouvement Social, 2016, n²54, p. 133-144.
} 
Voilà, c'est le choix qui a été fait, et c'est bien ce qui a été fait à l'époque par le ministre des sports qui était Marie-George Buffet. (Entretien du 4 septembre 2014)

\section{Tableau 1 : Couples d'opposition activés par les négociations dans la branche sport}

\begin{tabular}{|r|l|}
\hline champ d'application & $/$ champ sportif \\
animation socioculturelle & $/$ animation sportive \\
secteur marchand & $/$ secteur non marchand \\
secteur associatif & $/$ secteur commercial \\
pratiques de loisir & $/$ pratiques de compétition \\
économie sociale et solidaire & $/$ entreprises \\
amateurisme & $/$ professionnalisme \\
\hline
\end{tabular}

Ces oppositions, bien loin d'être nouvelles dans le champ sportif, trouvent dans les négociations de branche une nouvelle scène d'expression où les différentes organisations, salariales et patronales, confrontent leur propre définition du sport et s'affrontent en séance afin de promouvoir leur point de vue et leurs représentations. Les premiers travaux voient ainsi le secteur des activités de remise en forme et de fitness, regroupées sous la bannière du Syndicat national des exploitants d'installations sportives et de services sportifs (SNEISS), contester par recours le champ d'application de la CCNS, en quitter les négociations et demander finalement leur rattachement à une autre convention collective ${ }^{26}$ en présentant cet argument symptomatique des rapports de force en présence :

Nous y gagnons : - De dépendre d'une convention qui présente la souplesse requise pour la gestion du personnel dans le monde des loisirs. - De ne pas dépendre d'une convention collective, celle du Sport, entièrement dominée côté employeurs par les représentants du secteur associatif et subventionné. ${ }^{27}$

Selon une stratégie inverse à celle de l' « exit » dont on rappelle qu'elle a également tenté pour un temps le football professionnel, la Fédération nationale des entreprises des activités

\footnotetext{
${ }^{26}$ Le SNEISS disparu depuis, rejoint à ce moment-là, la Convention collective nationale des espaces de loisirs et attractions culturelles (CCNELAC). Il convient de rappeler que l'incertitude a pesé jusqu'au bout puisque le SNEISS ne s'est officiellement retiré du jeu par lettre recommandée qu'à la veille de la signature par toutes les parties prenantes de la convention permettant l'arrêté d'extension.

${ }^{27}$ Souligné par nos soins. Source : courrier du 5 décembre 2000 signé du président du SNEISS, archives de la CCNS déposées au CoSMoS.
} 
physiques de loisir (FNEAPL) demande depuis 2010, sans y parvenir jusqu'ici ${ }^{28}$, à intégrer la CCNS au titre du monopole qu'ils prétendent détenir en tant que «la seule organisation professionnelle représentant les entreprises des activités physiques de loisirs $»^{29}$. Touchant quasi systématiquement à des critères qui engagent la définition même du sport et des formes de monopole qui en découlent, ces entreprises de délimitations du champ d'application questionnent plus généralement le sens des limites propre au champ sportif ${ }^{30}$ et rejaillissent d'une manière renouvelée à travers les problématiques de travail et d'inscription des emplois dans un cadre conventionnel stable.

\section{Encadré 2 : le champ d'application de la CCNS, quelle définition du sport ?}

Avant même de réunir l'ensemble des partenaires sociaux et ouvrir les négociations sur le contenu même de la convention collective, les représentants des employeurs doivent ensemble en fixer le champ d'application, c'est-à-dire le périmètre des structures et entreprises qui seront concernées par le texte conventionnel. Dès 1996, la sous-direction de la négociation collective du Ministère du travail et des affaires sociales s'attache à animer des discussions avec les organisations professionnelles de l'animation socio-culturelle et celles du sport, via le CNOSF. Il s'agit en effet de «lever les ambiguïtés qui subsistent sur les frontières animation socioculturelle/sport et à l'intérieur du champ sport, les frontières entre sport associatif et sport commercial $»^{31}$. Dans une note manuscrite commentant la première réunion entre organisations employeurs, l'animateur soulignait la ferme résolution du CNOSF à embrasser tout le sport, sous toutes ses formes: «Sa position à l'heure actuelle reste la suivante: négocier une convention collective du sport qui engloberait les 3 secteurs, c'est-à-dire associatif, commercial et haut niveau $»^{32}$. L'extrait d'entretien ci-dessous, mené avec l'un des premiers négociateurs du CNOSF revient sur ces moments inauguraux et sur les problèmes auxquels renvoie la délimitation des frontières du champ d'application.

«La première chose qui est abordée, au niveau de la commission mixte paritaire, c'est le champ d'application. C'est-à-dire bah qui va devoir s'appliquer cette convention collective quoi. Donc pour nous ça semblait évident, le

\footnotetext{
${ }^{28}$ Les règles conventionnelles prévoient en effet que l'intégration d'une nouvelle organisation employeur ne peut s'envisager qu'à la condition d'un vote unanime, le CoSMoS ayant marqué jusqu'ici son désaccord. Le seul recours possible dans ce cas consiste à demander une enquête de représentativité auprès de la Direction générale du travail qui tranche en dernier ressort.

${ }^{29}$ Source : dossier de dépôt de candidature remis en séance de la commission mixte paritaire du 20 juin 2014.

${ }^{30}$ DEFRANCE, Jacques. 2012. «L'autonomie d'un champ ou le sens des limites », op. cit., p. 321.

${ }^{31}$ Sous-direction de la négociation collective, bureau des conventions collectives et des conflits collectifs du travail, courrier adressé aux organisations patronales de l'animation socio-culturelle et du sport, daté du 7 mai 1996. AN cote 20111081/68.

${ }^{32}$ AN cote $20111081 / 68$.
} 
mouvement sportif, on commençait à avoir des discussions avec le sport pro parce qu'on souhaitait qu'il soit là dans son application, le sport commercial, il y avait le SNEISS qui était là, donc normalement le sport commercial devait aussi rentrer dans ce champ. Sur la grande majorité il n'y a pas eu de souci mais après on arrivait les derniers. Bon. Et une convention et sur l'application d'une convention, c'est aussi des enjeux en terme de représentativité par rapport au Ministère du travail, c'est des enjeux financiers etc., etc., sur la formation professionnelle et il a été en fait très difficile et c'est pas fini, ça continue aujourd'hui d'ailleurs, de définir le champ d'application. Parce que l'animation revendiquait qu'un certain nombre de secteurs, que nous on trouvait sportif, était de l'animation, le tourisme social revendiquait un certain nombre de choses, l'équitation était déjà dehors puisqu'ils avaient leur propre convention collective, le golf était déjà dehors puisqu'il avait sa propre convention collective, le sport pro avait négocié des accords sectoriels de la charte du footballeur professionnel qui existait déjà et cette négociation en fait elle n'a abouti qu'en, attends je te dis...2001, c'est-à-dire que l'accord sur le champ d'application, c'est-à-dire l'article 1 de la convention collective c'est 2008..., non attends je dis des bêtises, 1998-2001 et on tombe à peu près d'accord. Donc on est sur 4 grands secteurs hein, qui comprend aussi la formation professionnelle et puis tout ce qui est évènementiel...mais quand je te disais rien n'est vraiment réglé, on a encore aujourd'hui des secteurs d'activités où on se bat. Par exemple, tu vas prendre des activités comme le yoga, les échecs, la danse, on est encore aujourd'hui en bisbille avec l'animation. L'animation, là, vient de créer, est en train de créer un certificat de qualification professionnelle danse qu'on conteste parce que la danse bénéficie d'un agrément sport donc on souhaite qu'elle reste sur le champ sport, la culture de son côté dit non, non, et non, la danse c'est chez nous, donc on est encore...c'est marginal aujourd'hui par rapport aux négociations de départ mais bon ça était et ça reste quelque fois source de problèmes et de conflits. (Entretien du 18 juillet 2012). »

La question des organisations susceptibles d'entrer ou non dans le champ d'application n'est n'est par conséquent pas neutre, loin s'en faut : elle engage avec chaque acteur une vision particulière du sport et contribue à fixer les frontières de ce qui peut être défini comme tel. Déterminer où commence et finit le sport revient par la force des choses à désigner qui en sera le ou les patrons et par incidence qui pourra participer à l'élaboration d'un droit du travail sportif conventionnel taillé sur mesure autant que faire se peut. La CCNS devient du même coup un lieu d'affrontements et de positionnements relatifs au monopole d'exercice et de représentation du sport, chaque acteur prétendant détenir au nom de l'organisation qu'il représente tout ou partie de celui-ci. L'observation ethnographique répétée des séances montre que les assertions explicitant les prises de positions, exacerbées par le contexte des prises de rôle, visent souvent à rappeler au préalable les positions depuis lesquelles elles s'expriment. Les préambules du genre «nous avons vocation à représenter tout le sport, sous toutes ses formes » (organisation patronale, CMP du 14 avril 2015) ou encore «vous rassemblez le sport pro, mais le sport associatif de base, c'est nous » (organisation patronale, CMP du 13 février 2013), «les employeurs de l'économie sociale et solidaire sont en ce moment pire que le MEDEF » 
(organisation salariée, CMP du 5 décembre 2012), «je suis employeur mais pas patron, je n'ai pas d'actions en bourse » (organisation patronale, CMP du 23 janvier 2014), «l'enjeu est que le sport ne soit pas aux mains de structures capitalistiques mais bien aux mains des associations » (organisation salariée, CMP du 15 mai 2014) marquent les territoires des uns et des autres et rappellent que l'unité prétendue du sport reste largement une fiction que souligne l'un des négociateurs du collège patronal :

On est parti dans cet environnement-là, moi je n'y étais pas à l'origine, sur un postulat qu'on a demandé d'accepter et ce postulat est le suivant : le sport est unique. Et à partir de là, on a mis à l'intérieur de ce postulat, un ensemble de composantes extrêmement différenciées, sans faire la démonstration que ces structures-là pouvaient fonctionner ensemble et faire vivre la notion de «le sport est unique». Donc belle construction intellectuelle, mais qui dans la vraie vie est pleine de failles. Et pour cause. Vous interrogez le monde du sport pro, ils vous diront mais nous, convention collective du sport, on vivait très bien chez nous, on a nos accords, foot, cyclisme, basket, etc., pas besoin de la convention collective du sport. (Entretien du 18 janvier 2013).

Très synthétiquement, les antagonismes se cristallisent de façon récurrente autour des couples d'opposition évoqués supra (voir tableau 1) et sur la capacité de chaque organisation à reprendre à leur compte ces lignes de partage. Du côté des employeurs, l'opposition la plus déterminante se fixe principalement autour de la capacité à représenter toutes les structures dans leur diversité : le CoSMoS prétendant parler pour tout le sport $^{33}$ mais étant renvoyé fréquemment au sport professionnel et/ou commercial ; le CNEA se positionnant résolument sur le domaine de l'économie sociale et solidaire pour toutes les structures sportives, à but lucratif ou non, mais étant régulièrement réduit au seul secteur associatif. Prises dans une lutte de représentation hégémonique, les deux organisations sont perpétuellement condamnées à produire la preuve de leur capacité à embrasser l'ensemble des activités et formes sportives et à les représenter, et se reprochent du même coup mutuellement leur inaptitude à le faire effectivement ${ }^{34}$. Du côté du

\footnotetext{
${ }^{33}$ Les prétentions du CoSMoS à couvrir tout le sport se trouvent nettement exprimées sur la page de présentation du site web de l'organisation où l'on peut lire que «Le CoSMoS est l'organisation de TOUS les employeurs du sport. Il accompagne ses adhérents dans leur rôle d'employeur et les représente dans les négociations de la Convention Collective Nationale du Sport » et que «Le CoSMoS est la première et l'unique organisation représentant exclusivement l'ensemble des employeurs du sport, qu'ils soient issus du secteur associatif, commercial ou du sport professionnel. ». Source: http://cosmos.asso.fr/rubriquage/section/53, consulté le $1^{\text {er }}$ décembre 2015.

${ }^{34}$ Ainsi le président du CoSMoS rappelait en entretien « je suis le président du CoSMoS donc je le disais, aujourd'hui, je me dois d'être garant d'une certaine unité de la branche et du mouvement sportif. Au sens large, parce qu'il n'y a pas que les fédérations, il y a aussi des entreprises, y a aussi plein d'autres structures. » (Entretien
} 
collège employés, le jeu s'est longtemps fixé autour d'une partition entre centrales syndicales généralistes (FO, CGT, CFDT, CGC, CFTC) et les autres organisations, notamment catégorielles (CNES, UNSA, FNASS). Depuis les élections professionnelles et les mesures d'audience de la représentativité syndicale auxquelles elles ont donné lieu en $2013^{35}$, seule la FNASS représentant exclusivement les salariés du sport professionnel siège encore aux côtés des centrales syndicales habituelles ${ }^{36}$, si bien que la ligne de partage se réalise principalement autour la distinction observée entre le traitement réservé aux salariés ordinaires et celui exclusivement dédié aux salariés du secteur professionnel. Face à de l'incapacité initiale à définir ce qu'est un sportif professionnel au plan juridique ${ }^{37}$, c'est en effet l'usage sportif, notamment le niveau de jeu ou de pratique, qui détermine quels salariés relèvent du sport professionnel. Par commodité et prenant acte des pratiques en vigueur, ces derniers sont traités dans la convention collective dans un chapitre particulier, le chapitre 12, qui ouvre un régime dérogatoire au droit commun (recours aux CDD, grille de salaires spécifique,...) adopté au nom de la spécificité sportive et du maintien impératif de l'équité des compétitions ${ }^{38}$. C'est donc bien plus le clivage sport professionnel / sport non professionnel qui se trouve activé ici, mais l'on comprend par là même l'intérêt à aboutir à une définition restrictive du sport, centrée prioritairement sur ses dimensions compétitives. Ce découpage confère au demeurant une plus forte centralité au CoSMoS et au CNEA en évitant une dilution en de multiples organisations patronales aux intérêts variés et non nécessairement convergents, et favorise pour les organisations salariés une partition des prérogatives où les confédérations traditionnelles s'intéressent de manière privilégiée aux salariés ordinaires de la branche quand la FNASS se préoccupe exclusivement des sportifs professionnels.

du 9 décembre 2014). Parallèlement, le représentant du CNEA affirmait recueillir parmi ses adhérents des structures sportives professionnelles et non pas seulement associatives (entretien du 24 janvier 2013).

${ }^{35}$ Ces mesures d'audience font suite à la suppression de la représentativité irréfragable évoquée supra. Elles contribuent lors des élections professionnelles à apprécier le poids numérique effectif des organisations syndicales salariées. Au niveau d'une branche professionnelle, une organisation devait atteindre $8 \%$ des suffrages pour être considérée représentative et participer aux négociations de la branche. Concernant la branche professionnelle du sport, ces mesures ont conduit à l'éviction de deux organisations, la Confédération nationale des éducateurs sportifs et l'Union nationale des syndicats autonomes. Sur cette question, lire BEROUD, Sophie ; LE CROM, Jean-Pierre ; YON Karel. 2012. "Représentativités syndicales, représentativités patronales. Règles juridiques et pratiques sociales. Introduction », Travail et Emploi, 131, p. 5-22.

${ }^{36}$ Arrêté du 27 novembre 2013 fixant la liste des organisations syndicales reconnues représentatives dans la convention collective nationale du sport $\left(n^{\circ} 2511\right)$.

${ }^{37}$ On veut souligner par le qualificatif « initiale » que la situation a changé depuis l'adoption de la Loi $\mathrm{n}^{\circ} 2015$ $1541 \mathrm{du} 27$ novembre 2015 «visant à protéger les sportifs de haut niveau et professionnels et à sécuriser leur situation juridique et sociale » où une définition précise du sportif professionnel apparait pour la première fois. Cette définition suscite actuellement une intense réflexion de la part des négociateurs de la branche pour la prendre effectivement en compte.

${ }^{38}$ FLEURIEL S., 2016, «Le sport professionnel saisi par sa convention collective: genèse d'une définition singulière », op. cit. 


\section{III - Des effets durables sur l'ordre sportif}

Il serait tentant à ce stade du raisonnement, de conclure que c'est bien la branche professionnelle du sport qui se trouve imprégnée des catégories d'analyse de l'ordre sportif, et qu'en définitive, ce dernier s'en trouve assez peu affecté. Mais c'est ici négliger les effets tout à fait réels que peut produire le travail normatif de couverture conventionnelle, en tant que « force du droit ${ }^{39}$, sur les pratiques concrètes des agents concernés par les activités sportives, mais qui reste bien peu visible dans les analyses relatives à la structuration du champ sportif. Au-delà du texte conventionnel, ce sont toutes les activités des commissions thématiques ${ }^{40}$ qui se trouvent engagées dans le processus en produisant et régulant les pratiques de travail et qui viennent, en retour, imprégner durablement les catégories de l'entendement sportif, dans des domaines clés tels que l'hygiène et la sécurité, la prévoyance, les politiques de formation et d'emploi, et surtout la formation professionnelle.

C'est précisément sur ce dernier point qu'on peut prendre la mesure des brèches que le dialogue social dans le sport est venu ouvrir, au travers de la désignation de l'organisme collecteur des fonds de formation professionnelle continue (organisme paritaire collecteur agréé (par l'État) ou encore OPCA). Contribuant à geler durablement les négociations sur quasiment l'ensemble des sujets portés à l'ordre du jour, et exacerbant les positions de toutes les organisations, ce sujet s'est révélé suffisamment grave pour être considéré significatif des manières d'appréhender et de penser le sport au cours des négociations. Jusqu'en janvier 2012, deux organismes collecteurs se trouvaient désignés pour la branche sport et respectaient de manière très consensuelle la partition bipolaire interne à celle-ci avec le choix d'Agefos, OPCA interprofessionnel recommandé par le CoSMoS et celui d'Uniformation, spécialisé dans l'économie sociale et préféré du CNEA ainsi que d'une partie des organisations salariées, en vertu de leur forte implication historique dans le conseil d'administration. De sorte que les organisations sportives, dont celles du sport professionnel, adhérentes du CoSMoS prenaient

\footnotetext{
${ }^{39}$ Entendue comme « forme par excellence du pouvoir symbolique de nomination et de classement qui crée les choses nommées et en particulier les groupes. Il n'est pas trop de dire qu'il fait le monde social, mais à condition de ne pas oublier qu'il est fait par lui. », voir BOURDIEU P., 1986, « La force du droit: Eléments pour une sociologie du champ juridique », Actes de la recherche en sciences sociales, 64, 1, p. 3-19.

${ }^{40} \mathrm{Au}$ nombre de 7 : la commission mixte paritaire (ou commission nationale de négociation), la commission paritaire nationale de l'emploi et de la formation du sport, la commission paritaire nationale d'interprétation et de validation, la commission paritaire nationale de prévoyance, la commission paritaire nationale sport professionnel, la commission paritaire nationale de prévention, d'hygiène, de sécurité et de veille sanitaire, la commission paritaire nationale de dialogue social.
} 
1'habitude de verser leurs contributions à leur propre OPCA interprofessionnel ${ }^{41}$ et développaient des actions de formation en fonction des relations privilégiées entretenues avec ceux-ci, pendant que les organisations adhérentes du CNEA fléchaient préférenciellement leurs fonds vers Uniformation.

Mais à partir du $1^{\text {er }}$ janvier 2012, cet équilibre, initialement inspiré d'une double désignation pragmatique permettant à toutes les organisations de faire l'économie d'un conflit, se trouve totalement remis en question par une modification substantielle du droit qui oblige dès lors à ne désigner plus qu'un seul OPCA par branche professionnelle. Actant jusqu'ici une polarisation des désignations observée selon des clivages internes, cette disposition réveille des enjeux puissamment conflictuels pour déterminer quel pôle, structures traditionnelles contre économie sociale, va emporter la mise, et par ricochet quelles conceptions de la formation professionnelle continue vont être adressées aux salariés de la branche. La signature de l'avenant 62 du 5 juillet 2011 ne laisse à cet égard a priori aucun doute sur l'état des rapports de force : le CNEA et les organisations de salariées désignent Uniformation à la majorité, et contre l'avis du CoSMoS (soit 9 organisations sur 10), puis demandent l'extension de l'arrêté pour son application généralisée auprès des employeurs de la branche ${ }^{42}$. Contre toute attente, ou plus vraisemblablement en vertu d'interventions politiques au plus haut niveau de l'Etat ${ }^{43}$, le Ministère du travail refuse l'extension et retarde l'échéance de son application, arguant par la voie de la Direction générale du travail que :

[...] Or en l'espèce, l'analyse du champ d'application de la convention collective du sport, fait apparaître que cette branche est composée de deux activités distinctes, les activités sportives à but lucratif d'une part, et les activités sportives à but non lucratif d'autre part.

Deux organisations professionnelles sont représentatives sur le champ d'application de la convention collective nationale du sport : le CNEA et le COSMOS. Le CNEA se limitant aux activités à but non lucratif de la branche du sport, il ne peut être représentatif

\footnotetext{
${ }^{41}$ En toute rigueur, il faudrait souligner qu'en plus d'Agefos, le second organisme collecteur interprofessionnel, Opcalia intervient également dans le processus en étant choisi par quelques structures du sport professionnel en dépit de la recommandation faite par le CoSMoS.

${ }^{42}$ La procédure d'extension consiste à rendre applicable, par arrêté ministériel ou interministériel, l'accord collectif de branche à tous les salariés et employeurs compris dans son champ d'application. L'accord étendu s'applique ainsi aux entreprises qui ne sont pas adhérentes à l'une des organisations patronales signataires.

${ }^{43}$ On regrette ici l'absence de matériaux permettant d'étayer cette supposition, mais ces événements restent trop récents pour qu'on puisse en trouver trace dans les archives. Plusieurs de mes informateurs ont évoqué cette hypothèse pour expliquer l'attitude pour le moins inattendue des services de la DGT, sans néanmoins pouvoir fournir d'éléments tangibles permettant d'en valider la pertinence.
} 
des activités sportives à but lucratif, qui sont incluses dans le champ d'application de l'avenant. Il ressort de ses statuts qu'il a vocation à représenter les « employeurs associatifs et autres organismes de l'économie sociale, gérant de façon désintéressée des activités éducatives, sportives, culturelles, scientifiques, sociales, de tourisme, de formation, de protection de l'environnement. »

Or, cet avenant, qui porte sur l'ensemble du champ d'application de la convention collective nationale du sport a été signé par le seul CNEA.

Telles sont les raisons du présent refus d'extension qui ont été présentées à la souscommission de l'extension.». ${ }^{44}$

Ce n'est pas tant la position en soi adoptée par le Ministère du travail que le motif pour lequel il justifie son choix qui intéresse ici : reprenant à son compte la division binaire du monde sportif entre structures lucratives et structures non lucratives, il apporte certes un soutien non négligeable au CoSMoS, mais le place également dans une situation embarrassante en le cantonnant à une seule sphère (les activités sportives à but lucratif) en dépit de tous les efforts que l'organisation patronale déploie pour s'imposer comme le porte-parole de toutes les organisations sportives. A contrario, bien que défavorable au CNEA, cet avis lui offre une occasion de réaffirmer son positionnement selon lequel il représente "des intérêts des employeurs de toutes les formes de structures sportives et de toutes les facettes d'expression de l'activité sportive, en ayant comme préoccupation première l'intérêt général et le refus des corporatismes $^{45}$. » Porté en recours devant le Conseil d'Etat en juin 2012 par les organisations salariées, dont le choix d'Uniformation était unanime et explicite, le contentieux prend en définitive une tournure défavorable au Ministère et par conséquent au CoSMoS, par un avis rendu le 16 mai 2014 qui annule pour abus de pouvoir la décision de ne pas procéder à l'extension et qui constate accessoirement que :

Si les structures à but non lucratif sont plus particulièrement représentées par une organisation d'employeurs spécifique, le CNEA, et si le ministre chargé du travail fait valoir les spécificités séparant la pratique du sport à titre amateur et à titre professionnel, il ne ressort pas des pièces du dossier que les activités sportives à but non lucratif, qui ne coïncident d'ailleurs pas avec les activités sportives exercées par des amateurs,

\footnotetext{
${ }^{44}$ Courrier du Directeur Général du Travail, Jean-Denis Combrexelle adressé au CNEA daté du 3 avril 2012 en réponse à leur communiqué du 9 décembre 2011.

${ }^{45}$ Communiqué du CNEA du 9 décembre 2011 qui fait suite au refus d'extension susmentionné et provoque la réponse du directeur général du travail en date du 3 avril 2012.
} 
présentent des particularités telles qu'elles puissent être regardées comme constituant une branche d'activité distincte des autres activités sportives. ${ }^{46}$

Parce qu'elle est opératoire et signifiante dans le champ sportif, la division entre secteurs lucratif et non lucratif s'est ainsi trouvée portée à l'agenda de la branche professionnelle du sport. Pour les mêmes raisons, c'est-à-dire en tant que catégorie pratique, sa mise en débat sur la scène conventionnelle a produit par ricochet des effets marquants dans le champ sportif. Une fraction des employeurs adhérents du CoSMoS, peu satisfaits de la tournure des évènements et peu disposés à abandonner leur OPCA historique, exercent alors de fortes pressions sur la direction de l'organisation patronale et manifestent un mécontentement qui irrigue jusque dans les couloirs du CNOSF. Bien que juridiquement différenciées, les deux organisations continuent d'entretenir des relations étroites, notamment par le biais d'une convention financière qui permet d'amener le CoSMoS à l'équilibre budgétaire et d'éviter le déficit chaque année. Cette situation créée les conditions d'une crise interne à la direction du CoSMoS qui débouche sur sa démission collective en mai 2013 et l'organisation d'élections pour la désignation d'une nouvelle équipe de direction. S'il n'est pas absolument certain que crise des OPCA et crise de gouvernance interne au CoSMoS soient corrélées, - le président nouvellement élu, Philippe Diallo, s'en défendant, la stricte coïncidence des agendas jette le trouble. Alors que l'affaire des OPCA, suspendue au jugement du Conseil d'Etat, semble durablement enlisée et ne trouve pas de solution conventionnelle viable, la direction du CoSMoS est entièrement renouvelée et désigne pour sa présidence un représentant notoire du football professionnel via l'UCPF. Pour un temps hostile à leur entrée dans la CCNS, 1'UCPF en devient objectivement un rouage central par le jeu de la représentation patronale :

D'abord effectivement, moi, je suis issu du sport professionnel donc, mon arrivée à la tête du CoSMoS avec son créateur au CNOSF, avec beaucoup de fédérations, beaucoup d'associations, le mouvement olympique, beaucoup de sections amateurs, en soi est une surprise. Et, et, et, une surprise doublée de quelque chose qui était relativement improbable compte tenu des rapports de force en termes de nombres. Puisque le sport professionnel a une économie développée mais finalement regroupe assez peu de membres. Donc... c'est pour ça qu'il était relativement peu probable que quelqu'un issu du sport pro, et j'ai envie de dire, au sein du sport pro, du football pro, qui pour certains garde une image un peu sulfureuse de sports business, de dérives diverses et variées. Et

\footnotetext{
${ }^{46}$ Source : Conseil d'état. $1^{\text {ère }}$ sous-section jugeant seule. 1405 2014. № 359877 Inédit au recueil Lebon.
} 
quelqu'un issu de cet univers, arrive à la tête du CoSMoS, je pense que ça a dû en surprendre et étonner plus d'un. (Entretien du 9 décembre 2014 avec le président du CoSMoS, directeur général de l’UCPF)

Ce revirement de situation semble ainsi marquer une évolution stratégique : tout se passe comme si à défaut de pouvoir se sortir des négociations conventionnelles, il valait mieux en prendre le contrôle. De fait, la délégation du CoSMoS qui se présente désormais à la table des négociations en commission mixte paritaire a sensiblement changé de profil. Alors que la précédente équipe laissait une large place à la représentation fédérale, la nouvelle délégation est majoritairement constituée de représentants du sport professionnel (voir tableau 2: évolution de la composition de la délégation du CoSMoS au tournant des élections de 2013) ; faisant conclure à l'ensemble des négociateurs des deux collèges qu'il s'agit bien là d'une reprise en main du CoSMoS par le sport professionnel.

En dépit du renouvellement de la direction du CoSMoS et de ses efforts pour renverser les orientations retenues par les partenaires sociaux en commission, le jugement du conseil d'Etat sonne comme un coup de tonnerre et débouche, après la signature d'un nouvel avenant majoritaire, sur l'avis d'extension désignant effectivement Uniformation comme OPCA de branche ${ }^{47}$ et contraignant le patronat sportif à revoir sa stratégie de formation à destination des salariés et à réorganiser autrement les relations très privilégiées qu'il entretenait jusqu'ici avec ses collaborateurs historiques de la formation professionnelle. Au-delà de l'affaire en ellemême, ce cas montre en quoi le processus de normalisation du travail sportif peut venir affecter durablement l'ordre sportif et en modifier les pratiques de fonctionnement. Dotées d'une réelle force de contrainte, les activités conventionnelles travaillent de fait la réalité sportive et en modifient la perception.

Tableau 2 : évolution de la composition de la délégation du CoSMoS en commission mixte paritaire au tournant des élections de 2013

\begin{tabular}{|l|c|c|}
\hline \multicolumn{1}{|c|}{ Représentants (de) } & Jusqu'en 2013 & Après 2013 \\
\hline Fédérations & 6 & 1 \\
\hline Structures du sport professionnel & 2 & 4 \\
\hline Autres structures (UCPA, Vert Marine) & 2 & 1 \\
\hline Salarié(s) du CoSMoS & 1 & 2 \\
\hline
\end{tabular}

\footnotetext{
${ }^{47}$ Il s'agit de l'avenant 97 relatif à la désignation de l'OPCA de branche étendu par arrêté du 21 juillet 2015 (paru
} au JO du 29 juillet 2015). 


\section{Conclusion : ce que le dialogue social fait au sport}

Que fait en définitive le dialogue social au sport? L'effet le plus probant est sans doute d'avoir contraint les dirigeants sportifs à se penser en patrons, dotés d'instances représentatives, nécessairement distinctes de leurs tutelles traditionnelles (fédérations, CNOSF) et invités à travailler avec des interlocuteurs relativement nouveaux pour eux. Si le CoSMoS n'est pas seul dans la branche du sport, loin s'en faut, il est le seul à avoir dû opérer cette révolution de nature toute copernicienne l'obligeant de se décentrer d'une vision proprement sportive du monde sportif, quand les autres organisations étaient par avance rompues à une appréhension du travail par son droit. Condamnés assez rapidement à décrypter leur environnement selon de nouvelles clés de lecture propres au droit conventionnel, ces nouveaux patrons dont les organisations représentatives restent plus généralement mal connues et peu étudiées ${ }^{48}$, ont dû composer avec les organisations syndicales pour redéfinir leurs manières de penser les plus viscéralement ancrées dans leurs habitudes dirigeantes. Les données d'enquête permettent alors de constater dans une perspective constructiviste que les catégories de classement et de compréhension des pratiques sportives ne sont jamais données en soi et renvoient bien en permanence à des luttes de définition qui prennent part sur les multiples scènes que déploie l'ordre sportif. La définition du sport engagée dans les pratiques n'est pas seulement discutée dans les instances sportives les plus visibles (fédérations, CNOSF, ligues professionnelles,...). Elle se trouve également durablement questionnée sinon aux confins du champ sportif, du moins dans ses régions les moins exposées aux regards, notamment dans la branche professionnelle du sport à partir de référentiels propres au monde du travail qui contribuent à déplacer la question, en faisant entrer d'autres interlocuteurs dans la danse, et à en modifier l'économie générale des interactions. Bien que ce déplacement soit rarement pris en considération dans les analyses portant sur la dynamique du champ, parce que probablement peu perceptible depuis ce point de vue, il ne produit pas moins d'effets pratiques sur celle-ci ; l'ordre sportif se trouvant mis en demeure de composer avec un ordre juridique parfois contraignant. Le patronat représenté dans la branche se confondant avec une partie des dirigeants sportifs, il importe en effet dans celle-ci ses propres catégories de l'entendement sportif et parvient à les imposer dans la discussion comme des clés

\footnotetext{
${ }^{48}$ Jean Saglio notait à ce propos dès 2006 que la « question de l'employeur et des organisations d'employeurs reste le trou noir de nos approches », constat que confirmait encore Michel Offerlé dans ses récents travaux sur le MEDEF. Voir SAGLIO, Jean. 2006, op. cit., p. 240 et OFFERLE, Michel. 2013, Les patrons des patrons: histoire du Medef, Paris, O. Jacob, 364 p.
} 
de lecture pertinentes. Appropriées puis travaillées par tous les acteurs de la branche, celles-ci trouvent de nouvelles traductions normatives et régulatrices qui viennent s'inscrire dans le droit conventionnel comme réponses aux difficultés et clivages observés dans le champ sportif. Par retour, elles diffusent dans celui-ci autant comme nouvelles contraintes objectives que comme expérience doxique à un ordre social ${ }^{49}$ cherchant à normaliser le travail sportif. Ce n'est sans doute là pas le moindre des effets.

${ }^{49}$ BourdieU, Pierre. 2012. Sur l'État: cours au Collège de France (1989-1992), CHAMPAGNe, Patrick ; LENOIR, Rémi ; POUPEAU, Franck (dirs.), Paris, Seuil : Raisons d'agir. 